\title{
New power decoupling strategy for grid-connected inverter under weak power grid
}

\author{
Rong-Jong $\mathrm{Wai}^{1 *}$, and Yu Wang ${ }^{1}$ \\ ${ }^{1}$ Department of Electronic and Computer Engineering, National Taiwan University of Science and Technology
}

\begin{abstract}
This study analyzes the power coupling in a weak power grid, and designs a novel decoupling method for the coupling power with multi harmonic frequency on the dc bus. By introducing a ratio factor for the dc-bus capacitors, the decoupling ability can be further improved. Moreover, the sliding-mode controller will enhance the system robustness and be adaptive on the varied condition of the power coupling. The proposed control strategy will be effective under the ideal power grid or even the non-ideal power grid. In addition, numerical simulations are provided to verify the effectiveness of the proposed method.
\end{abstract}

\section{Introduction}

Inverters have been widely used in the uninterruptible power supply (UPS), distributed generation (DG), and other small and medium-power applications. However, the inherent ripple power on the ac side of a single-phase inverter system leads to the voltage ripples with low-frequency harmonic components on the dc bus. The undesirable ripple voltage will degrade the system performance, reduce output inverter power quality, increase the loss of the inverter system, and shorten the life span of input power sources, e.g., photovoltaic (PV) panels or battery modules [1]-[3].

Since the passive power decoupling method has the disadvantages of low power density and low reliability, many researchers have proposed active power decoupling methods to restrain the ripple voltages on the dc bus in recent years. The control strategies for active decoupling methods are mainly classified into the open-loop control [4]-[5] and the closed-loop control [6]-[7]. Although the open-loop control method is relatively simple, it is sensitive to the parameter variations of the energy storage component, and is difficult to achieve the accurate power decoupling. Mellincovsky et al. [6] introduced a decoupling method with a bidirectional buck-boost circuit paralleled with the dc-bus capacitor as the decoupling topology. The decoupling controller in [6] utilized a proportional-integral (PI) controller for stabilizing the dc-bus voltage while a multi-resonant controller was paralleled for suppressing the ripple voltages. Liu et al. [7] combined the power decoupling circuit with the power-factor-correction (PFC) circuit. The circuit in [7] can achieve the high power factor, the wide output-voltage range, and the ripple-power decoupling without using electrolytic capacitors. Unfortunately, all these above methods require additional energy storage devices to absorb the ripple power. Although the dc-bus capacitance can be reduced to a certain extent, additional energy storage devices increase the system volume and manufacturing cost.

In the past, Tang et al. [8] presented an active power decoupling method by using a half-bridge inverter circuit and series split-capacitors. Because the split capacitors were used as the dc-bus capacitor, additional energy storage devices were not required. However, the effect of the capacitor parameter deviation was not considered. Yao et al. [9] proposed a new control method and discussed the effects of the capacitance mismatch on the power decoupling. In [9], each dc-bus capacitor voltage was forced to track a different dc offset and a double-line-frequency, rather than the sinusoidal line-frequency one. As can be seen from the experimental results in [9], the effectiveness of this decoupling circuit can be verified. Therefore, the structure of the half-bridge with series split-capacitors is adopted as the decoupling circuit in this study.

Basically, all these aforementioned power coupling methods were investigated under the assumption of an ideal power grid, and the corresponding control strategies were mostly designed for only dealing with the coupling power with twice fundamental frequency. With the increasing penetration of DG, the power grid gradually presents the property of a weak power grid, and the magnitudes of low-frequency harmonics at the point of common coupling (PCC) are also correspondingly high [10]. When the power grid is not ideal, the instantaneous power coupling on the dc bus is not only a second-order one. Therefore, it is necessary to proposed an adaptive method for decoupling the correspondingly coupled

*Corresponding author: rjwai@mail.ntust.edu.tw 
power automatically. This study is mainly organized into five sections. Following the introduction, the problem of power coupling in non-ideal power grid is briefly described in Section 2. In Section 3, an adaptive power decoupling method under a weak power grid is proposed to achieve a better power decoupling. In Section 4, the corresponding numerical simulations are provided to verify the effectiveness of the proposed method. Finally, some conclusions are given in Section 5.

\section{Power Coupling In Non-ideal Power Grid}

As the penetration rate of distributed generation systems increases, the power grid gradually presents a weak power-grid status. High power-grid impedance and different harmonic sources will lead to the distortion on the grid voltage. Therefore, the voltage at the point of common coupling (PCC) contains more low-frequency harmonics [10]. By taking the 3rd harmonic component as an example, the expression of the grid voltage and the grid current can be represented as

$$
\left\{\begin{array}{l}
u_{\mathrm{g}}(t)=\sqrt{2} U_{\mathrm{g}} \sin (\omega t)+\sqrt{2} k U_{\mathrm{g}} \sin (3 \omega t) \\
i_{\mathrm{g}}(t)=\sqrt{2} I_{\mathrm{g}} \sin (\omega t+\varphi)
\end{array}\right.
$$

where $U_{\mathrm{g}}$ and $I_{\mathrm{g}}$ are the corresponding effective values of the grid voltage and current, respectively; $\omega$ is the grid frequency; $k$ represents the percentage of 3rd harmonic component; $\varphi$ is the phase angle. Therefore, the instantaneous power of (1) can be rewritten as

$$
\begin{aligned}
p_{\mathrm{g}} & =2 I_{\mathrm{g}} U_{\mathrm{g}} \sin \omega t \sin (\omega t+\varphi)+2 k I_{\mathrm{g}} U_{\mathrm{g}} \sin 3 \omega t \sin (\omega t+\varphi) \\
& =I_{\mathrm{g}} U_{\mathrm{g}} \cos (\varphi)-\underbrace{I_{\mathrm{g}} U_{\mathrm{g}} \cos (2 \omega t+\varphi)+k I_{\mathrm{g}} U_{\mathrm{g}} \cos (2 \omega t-\varphi)}_{\text {2nd Ripple Power }} \\
& -\underbrace{k I_{\mathrm{g}} U_{\mathrm{g}} \cos (4 \omega t+\varphi)}_{\text {4th Ripple Power }}
\end{aligned}
$$

Obviously, in (2), there exists not only a second-order ripple power, but also a fourth one, which will lead to a 4th ripple voltage on the dc bus. The 4th ripple voltage, which will be multiplied by the phase-locked-loop (PLL) measurement of the grid voltage, makes the grid current command to contain the correspondingly 5th harmonic component. If there are more low-frequency harmonics (e.g., 3rd, 5th, 7th) at the PCC, the dc-bus voltage will contain the corresponding voltage ripples (e.g., 4th, 6th, 8th). Accordingly, the grid current will contain higher-order harmonic components, which will further deteriorate the power quality at the PCC.

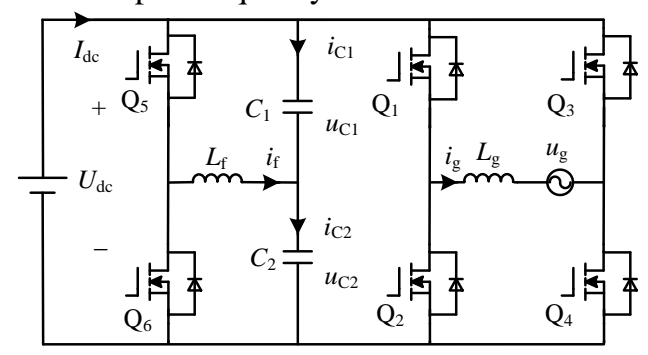

Fig. 1. Circuit framework of single-phase inverter with decoupling circuit.
The decoupling circuit of the half-bridge with series split-capacitors in [8] is adopted in this study. However, different from this decoupling structure, the values of the two series capacitors $\left(C_{1}\right.$ and $\left.C_{2}\right)$ are not the same. For improving the decoupling ability, this study introduces a ratio factor $l$ between $C_{1}$ and $C_{2}$, i.e., $C_{1}=l C_{2}$. The decoupling circuit can be depicted in Fig. 1.

By modulating the half-bridge inverter circuit, the voltage on each of two series capacitors can be expressed as

$$
\left\{\begin{array}{l}
u_{\mathrm{C} 1}=\frac{U_{\mathrm{dc}}}{2}+u_{\mathrm{C}}=\frac{U_{\mathrm{dc}}}{2}+U_{\mathrm{C}} \sin (2 \omega t+\theta) \\
u_{\mathrm{C} 2}=\frac{U_{\mathrm{dc}}}{2}-u_{\mathrm{C}}=\frac{U_{\mathrm{dc}}}{2}-U_{\mathrm{C}} \sin (2 \omega t+\theta)
\end{array}\right.
$$

where $\theta$ is the angle between grid voltage $\left(u_{\mathrm{g}}\right)$ and $u_{\mathrm{C}}$; $U_{\mathrm{C}}$ is the peak value of the sinusoidal components superimposed on the capacitor. Thus, the instantaneous power contributed by the decoupling circuit can be expressed as

$$
\begin{aligned}
p_{\mathrm{h}} & =p_{\mathrm{C} 1}+p_{\mathrm{C} 2}+p_{\mathrm{Lf}} \\
& =(l-1) \omega C_{2} U_{\mathrm{C}} U_{\mathrm{dc}} \cos (2 \omega t+\theta) \\
& +(l+1) \omega C_{2} U_{\mathrm{C}}^{2}\left[1-4(1+l) \omega C_{2} \omega L_{\mathrm{f}}\right] \sin (4 \omega t+2 \theta)
\end{aligned}
$$

As can be seen from (4), the power decoupling can be realized as long as the double-line-frequency power component in $p_{\mathrm{h}}$ and (2) are equal. By the same way, the 4th ripple power in $p_{\mathrm{h}}$ will further compensate the corresponding one generated by the 3rd harmonic of the power-grid voltage. When there are other higher-order harmonics at the PCC, the corresponding coupling power can be decoupled by adding higher-order ac components in (3). When the value of $l$ is fixed, the decoupling ability is only depended on the value of $U_{c}$. By equating the double-line-frequency component in (2) and (4), the relationship of $U_{\mathrm{c}}$ and phase angle can be derived as

$$
\left\{\begin{array}{l}
U_{\mathrm{c}}=\frac{U_{\mathrm{g}} I_{\mathrm{g}} \sqrt{(k+1)^{2}-4 k \cos ^{2} \varphi}}{(l-1) \omega C_{2} U_{\mathrm{dc}}}, l \neq 1 \\
\theta=\arctan \left(\frac{k+1}{1-k} \tan \varphi\right)
\end{array}\right.
$$

The implementation flowchart of the proposed power decoupling strategy can be depicted in Fig. 2. When the voltages across the series capacitors satisfy the relationships of (3) and (5), the power decoupling can be realized.

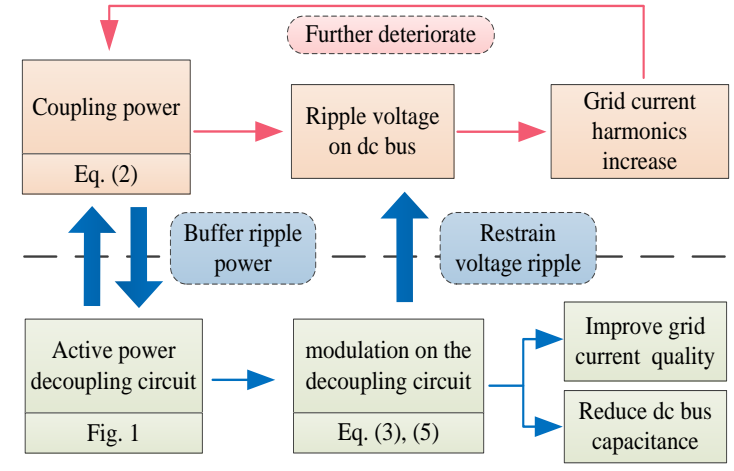

Fig. 2. Implementation flowchart of proposed adaptive power decoupling strategy. 


\section{Adaptive Power Decoupling Under Weak Power Grid}

According to the analyses in Section 2, the power decoupling will be achieved when the voltages across the series capacitors satisfy the relationships of (3) and (5). However, it is open-loop control and it is difficult to achieve accurate decoupling by solving the expression in (5), especially when the parameter deviation occurs. What is more, when the power grid is non-ideal, the harmonic orders at the point of common coupling (PCC) are not fixed. Thus, it is hard to accurately know the specific orders of the coupling power. Therefore, the closed-loop control is required to further design.

In order to achieve the totally power decoupling, the decoupling circuit should provide the accurate decoupling power. However, the ripple voltages on the dc bus have multi-frequency harmonics and the frequency is not fixed depending on the different power grid conditions. Thus, the traditional control method (e.g., multi proportional resonant controller) will be hardly to achieve an accurate power decoupling under the non-ideal power grid. For achieving this goal, an adaptive power decoupling method is proposed, and the control block diagram is depicted in Fig. 3. By considering the superiority on the nonlinear robust control and the insensitivity of the parameter variations and external disturbance, a sliding-mode controller (SMC) [11] is adopted here. In this study, the SMC is divided into two parts. The first one is the baseline model design, which will specify the desired performance. According to the control scheme in Fig. 3, the system state equation without the parameter variations can be expressed as

$$
\ddot{u}_{\text {rip }}(t)=a_{\mathrm{n}} \dot{u}_{\text {rip }}(t)+b_{\mathrm{n}} i_{\mathrm{f}}^{*}(t)+c_{\mathrm{n}}
$$

where $a_{\mathrm{n}}=-k_{\mathrm{p}} K_{\mathrm{pwm}} / L_{\mathrm{f}} ; b_{\mathrm{n}}=-l k_{\mathrm{p}} K_{\mathrm{pwm}} /\left[(1+l) C_{1} L_{\mathrm{f}}\right]$; $c_{\mathrm{n}}=i_{\mathrm{C} 2} l\left(L_{\mathrm{f}} s+k_{\mathrm{p}} K_{\mathrm{pwm}}\right) /\left(C_{1} L_{\mathrm{f}}\right) ; u_{\text {rip }}$ is the ripple voltage; $i_{\mathrm{f}}^{*}$ is the desired inductor current to be designed by the $\mathrm{SMC} ; k_{\mathrm{p}}$ is the proportional gain of the inner current loop; $K_{\mathrm{pwm}}$ represents the transfer function of the modulation to the output voltage of the half-bridge inverter; $l$ is the ratio factor of $C_{1}$ and $C_{2}$. Define a sliding surface as

$$
s_{l}(t)=\dot{e}(t)+k_{1} e(t)
$$

where $e(t)=u_{\text {rip }}^{*}-u_{\text {rip }}$ is the voltage tracking error, in which $u_{\text {rip }}^{*}=0$ is the desired ripple voltage; $k_{1}$ is a positive constant. The baseline model control law $\left(i_{\mathrm{fb}}\right)$ can be expressed as

$$
\left.i_{\mathrm{fb}}(t)=b_{\mathrm{n}}^{-1}\left[-a_{\mathrm{n}} \dot{u}_{\text {rip }}(t)-c_{\mathrm{n}}+k_{1} \dot{e}(t)\right)\right]
$$

By substituting (8) into (6) and using (7), one can obtain

$$
\begin{aligned}
\dot{s}_{l}(t) & =\ddot{e}(t)+k_{1} \dot{e}(t) \\
& =0-\ddot{u}_{\text {rip }}+k_{1} \dot{e}(t)=0
\end{aligned}
$$

Then, the second part is the curbing controller design for ensuring the robustness under the consideration of parameter variations and external disturbance. By considering the occurrence of system uncertainties, the system state equation in (6) can be rewritten as

$$
\begin{aligned}
\ddot{u}_{\text {rip }}(t) & =\left(a_{\mathrm{n}}+\Delta a_{\mathrm{n}}\right) \dot{u}_{\text {rip }}(t)+\left(b_{\mathrm{n}}+\Delta b_{\mathrm{n}}\right) \dot{i}_{\mathrm{f}}^{*}(t)+c_{\mathrm{n}}+\Delta c_{\mathrm{n}} \\
& =a_{\mathrm{n}} \dot{u}_{\text {rip }}(t)+b_{\mathrm{n}} \dot{i}_{\mathrm{f}}^{*}(t)+c_{\mathrm{n}}+d_{\mathrm{p}}(t)
\end{aligned}
$$

where $\Delta a_{\mathrm{n}}, \Delta b_{\mathrm{n}}$ and $\Delta c_{\mathrm{n}}$ are the parameter variations; and $d_{\mathrm{p}}(t)$ is the lumped uncertainty and can be expressed as

$$
d_{\mathrm{p}}(t)=\Delta a_{\mathrm{n}} \dot{u}_{\text {rip }}(t)+\Delta b_{\mathrm{n}} i_{\mathrm{f}}^{*}(t)+\Delta c_{\mathrm{n}}
$$

Here, the bound of $d_{\mathrm{p}}(t)$ is assumed to be given as

$$
\left|d_{\mathrm{p}}(t)\right|<\rho
$$

where $|\cdot|$ is the operator of an absolute value, and $\rho$ is a given positive constant. The curbing control law can be designed as

$$
i_{\mathrm{fc}}(t)=b_{\mathrm{n}}^{-1} \rho \operatorname{sgn}\left(s_{l}(t)\right)+b_{\mathrm{n}}^{-1} k_{2} s_{l}(t)
$$

where $\operatorname{sgn}(\cdot)$ is the sign function, and $k_{2}$ is a positive constant. Thus, the SMC law can be expressed as

$$
i_{\mathrm{f}}^{*}(t)=i_{\mathrm{fb}}(t)+i_{\mathrm{fc}}(t)
$$

By substituting (8), (13) and (14) into (10), one can obtain

$$
\dot{s}_{l}=-\ddot{u}_{\text {rip }}+k_{1} e(t)=-b_{\mathrm{n}} i_{\mathrm{fc}}-d_{\mathrm{p}}(t)
$$

In order to ensure the system stability, it should satisfy the following sliding condition:

$$
s_{l}(t) \dot{s}_{l}(t)<0
$$

According to (13) and (15), one can obtain

$$
\begin{aligned}
s_{l}(t) \dot{s}_{l}(t) & =s_{l}(t)\left[-b_{\mathrm{n}} i_{\mathrm{fc}}-d_{\mathrm{p}}(t)\right] \\
& \leq-s_{l}(t) b_{\mathrm{n}} i_{\mathrm{fc}}+\left|s_{l}(t)\right|\left|d_{\mathrm{p}}(t)\right| \\
& =-\rho\left|s_{l}(t)\right|-k_{2} s_{l}^{2}(t)+\left|s_{l}(t)\right|\left|d_{\mathrm{p}}(t)\right| \\
& =-\left|s_{l}(t)\right|\left(\rho-\left|d_{\mathrm{p}}(t)\right|\right)-k_{2} s_{l}^{2}(t)<0
\end{aligned}
$$

As can be seen from (17), the voltage tracking error $e(t)$ will converge to zero when time tends to infinity. In other words, the design of the inner inductor current by the SMC can indirectly force the voltage ripple to be zero.

\section{Numerical Simulations}

The proposed adaptive power decoupling method is verified by numerical simulations of $1.5 \mathrm{~kW}$ system in the MATLAB/Simulink software. In the simulations, the root mean square (RMS) value of the grid voltage is $220 \mathrm{~V}$, and the frequency of the grid voltage is $50 \mathrm{~Hz}$. The equivalent dc-bus capacitance is $65 \mu \mathrm{F}$, and the half-bridge filter inductor $L_{\mathrm{f}}$ is $2 \mathrm{mH}$. The parameters of the proposed

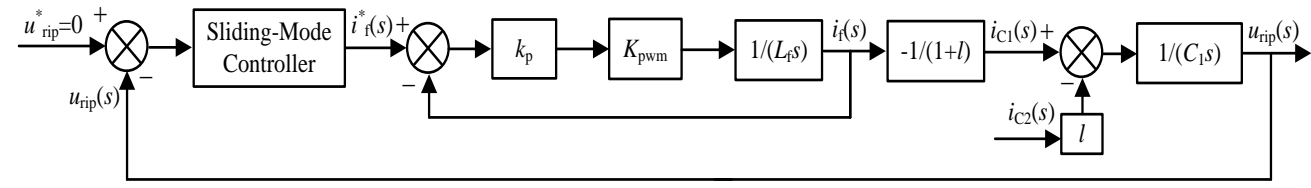

Fig. 3. Block diagram of adaptive power decoupling strategy. 
controller are given as $\eta=0.05, k_{1}=1000, k_{2}=1100$, and $\rho=10$.

Figure 4 shows the numerical simulations of the proposed adaptive power decoupling method under the changed condition from the ideal grid voltage to the distorted grid voltage. In Fig. 4(a), the power grid condition is changed at $1 \mathrm{~s}$, where the 3rd harmonic is $10 \%$, the 5 th harmonic is $10 \%$, and the 7 th harmonic is $5 \%$, and the total harmonic distortion (THD) value of the distorted grid voltage is measured as $14.47 \%$. Although the grid voltage has severe harmonics, the grid current has no obvious distortion. As can be seen from Fig. 4(c) and (d), the dc-bus ripple voltage is only about $2 \mathrm{~V}$ and the distorted power grid has almost no effect on it. That means the proposed method can achieve better power decoupling no matter the ideal power grid or the distorted power grid. The decoupling strategy can be adaptive on the varied condition of the coupling power.

Figure 5 shows the numerical simulations with and without the proposed adaptive power decoupling method under the distorted power grid. It can be seen from Fig. 5(a) and (b), comparing with the grid current before and after 1s, the grid current has an obvious distortion in distorted power grid because of the weak decoupling ability. In Fig. 5(c) and (d), the dc-bus ripple voltage has a significant decline in the proposed method. As can be seen from Fig. 5(d), the amplitude of the dc-bus ripple voltage in the proposed method are all less than $0.35 \mathrm{~V}$. However, without the proposed decoupling method, the amplitude of the 2nd harmonic is about $78 \mathrm{~V}$ and the other order harmonic are greater than $0.65 \mathrm{~V}$. Thus, the proposed method can achieve a better grid current because of the accurate power decoupling.

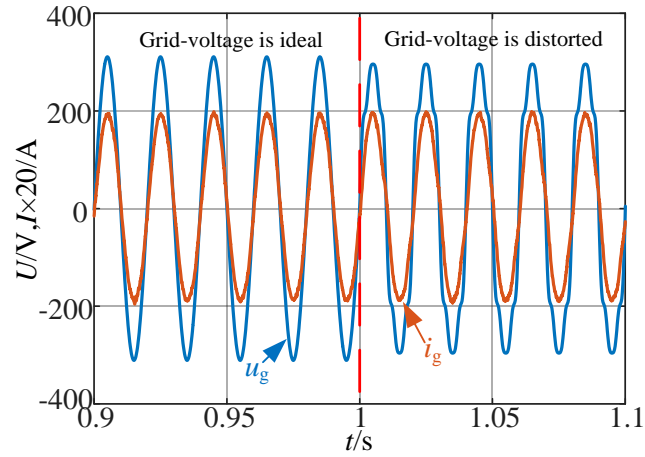

(a)

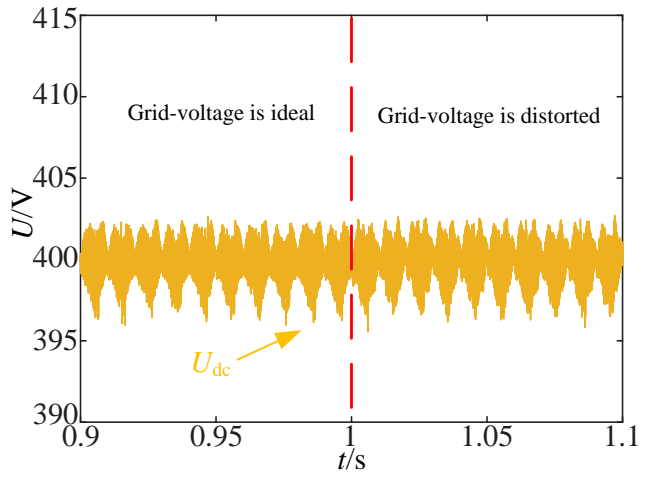

(c)
Figure 6 shows the numerical simulations of the proposed adaptive power decoupling method under the grid voltage and input power variation. In Fig. 6(a), the grid voltage drops $20 \%$ at 0.8 s. The dc-bus ripple voltage and the inverter output current are gradually stabilized within $0.05 \mathrm{~s}$, and the maximum dc-bus voltage fluctuation is about only 5V. In Fig. 6(b), the input power changed from 500 to $1000 \mathrm{~W}$. It can be seen that the dc-bus ripple voltage and the inverter output current are stabilized within $0.05 \mathrm{~s}$, and there is no obvious distortion Thus, the proposed method in this study has a good dynamic performance.

\section{Conclusions}

This study has presented a novel adaptive power decoupling method for alleviating the ripple power in a single-phase grid-connected inverter system under the condition of a non-ideal power grid. The system decoupling ability can be further improved by introducing a ratio factor for the two dc-bus capacitors. Moreover, the sliding-mode controller (SMC) can ensure the system to have robustness performance and be adaptive on different conditions of the power coupling. The design of the inner inductor current by the SMC can indirectly force the voltage ripple to be zero. Because of the accurate power decoupling, the equivalent dc-bus capacitance is only $65 \mu \mathrm{F}$, and the dc- bus ripple voltage can be restrained in a lower content (about $2 \mathrm{~V}$ ) whatever under the ideal power grid or a distorted power grid.

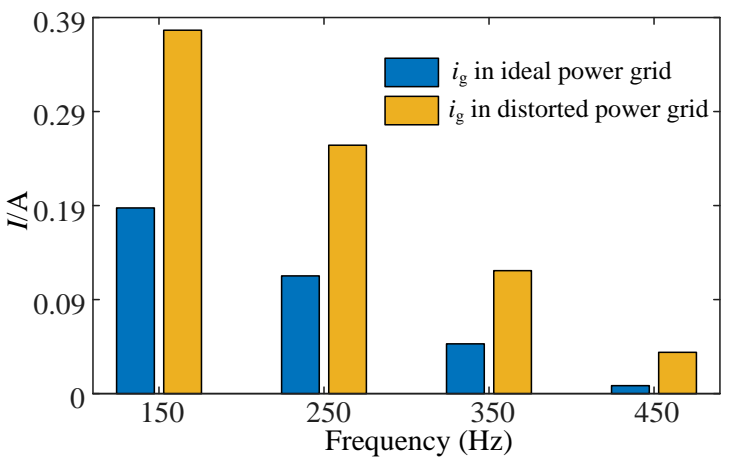

(b)

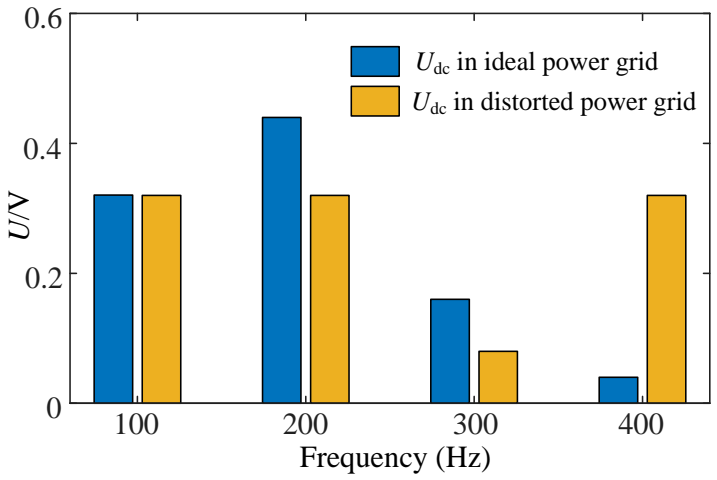

(d)

Fig. 4. Numerical simulations of proposed adaptive power decoupling method under changed power grid condition. (a) Grid voltage and grid current. (b) Spectrum of grid current. (c) Dc-bus voltage. (d) Spectrum of dc-bus voltage. 


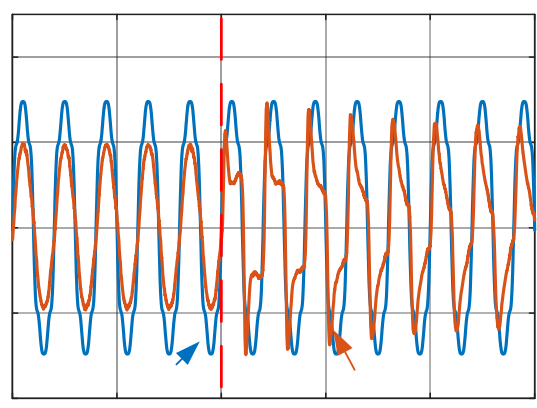

(a)

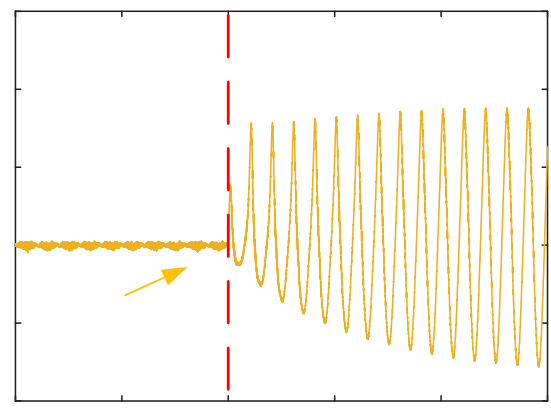

(c)

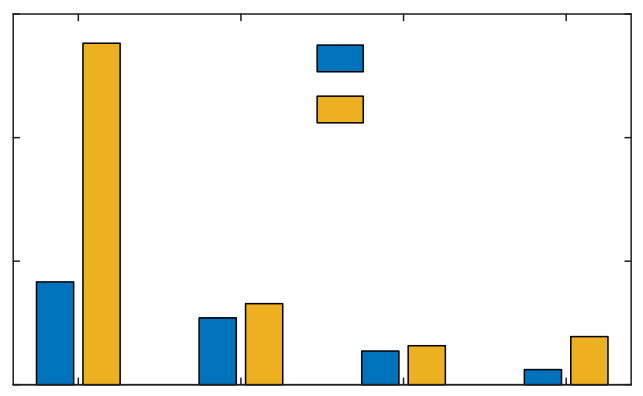

(b)

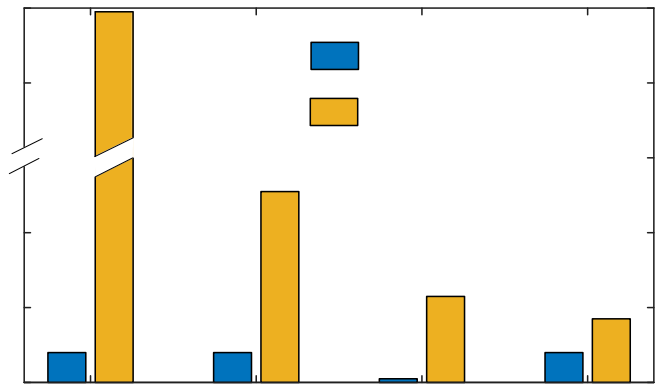

(d)

Fig. 5. Numerical simulations with and without the proposed adaptive power decoupling method under the distorted power grid. (a) Grid voltage and grid current. (b) Spectrum of grid current. (c) DC-bus voltage. (d) Spectrum of dc-bus voltage.

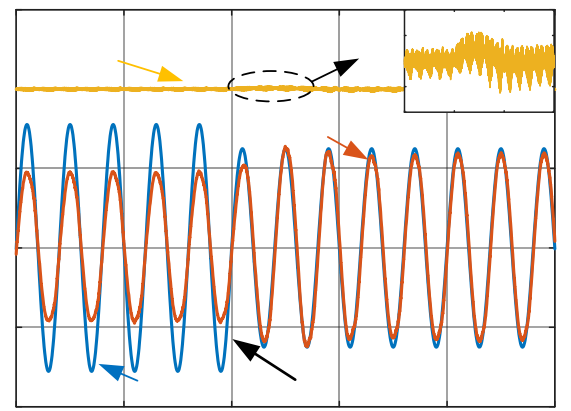

(a)

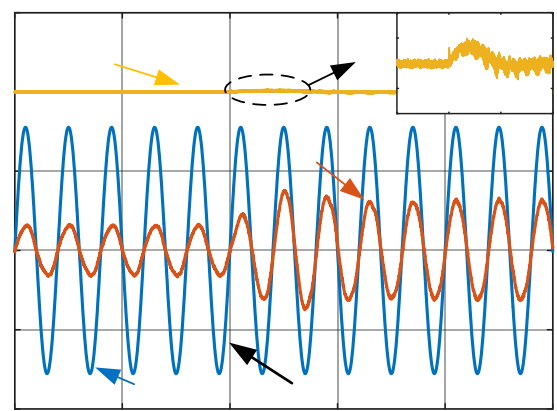

(b)

Fig. 6. Numerical simulations of proposed adaptive power decoupling method under grid voltage and input power variation. (a) Grid voltage drops. (b) Input power surges.

\section{References}

1. Z. Qin, Y. Tang, P. C. Loh, and F. Blaabjerg, IEEE J. Emerging and Selected Topics in Power Electron., 4, 15-25 (2016).

2. Y. Sun, Y. Liu, M. Su, W. Xiong, and J. Yang, IEEE Trans. Power Electron., 31, 4778-4794 (2016).

3. C. Y. Liao, W. S. Lin, Y. M. Chen, and C. Y. Chou, IEEE Trans. Power Electron., 32, 6544-6557 (2017).

4. G. Zhu, H. Wang, B. Liang, S. Tan, and J. Jiang, IEEE Trans. Ind. Electron., 63, 937-943 (2016).

5. I. Serban, IEEE Trans. Ind. Electron., 62, 4805-4813, (2015).
6. M. Mellincovsky, V. Yuhimenko, M. M. Peretz, and A. Kuperman, IEEE Trans. Ind. Electron., 64, 2015-2023 (2017).

7. Y. Liu, Y. Sun, M. Su, M. Zhou, Q. Zhu, and X. Li, IEEE Trans. Power Electron., (to be published).

8. Y. Tang, Z. Qin, F. Blaabjerg, and P. C. Loh, IEEE Trans. Power Electron., 30, 7060-7071 (2015).

9. W. Yao, P. C. Loh, Y. Tang, X. Wang, X. Zhang, and F. Blaabjerg, IEEE Trans. Power Electron., 32, 8419-8433 (2017).

10. J. Xu, Q. Qian, S. Xie, and B. Zhang, IEEE Applied Power Electronics Conference and Exposition (APEC) 1919-1925 (2016).

11. R. J. Wai and C. Y. Lin, IEEE Trans. Ind. Electron., 57, 3780-3792 (2010). 